\title{
AUTOMATED GALLERY LIGHTING SYSTEM AND SEEPAGE WATER CONTROL IN DAM
}

\section{ABDULGAFFAR DODDAMANI}

Assistant Professor, College of Horticulture Engineering and Food Technology Bagalkot, Karnataka, India

Now a day energy crisis in India is a critical issue. The present gallery lighting is controlled by manually and is leds to energy loss. The lighting system in the drainage gallery of the dam required during the maintenance work such as drilling and grouting the foundation. With the help of an automated lighting system, it reduces the power consumption of the lighting. This proposed system continuously monitors the pump's which are used to pump the collected water from the storage tank due to seepage. The main aim of theautomated gallery lighting system is to reduce the power consumption when there is no person movement in the gallery and continuously monitors the pump. The automated gallery lighting system will turn ON the LED with the help of proximity sensor which is attached to the LED set when there is a person present in the gallery otherwise the LED lights will be switched OFF. This system operates in two modes i.e. remote mode and automated mode. In the automated mode with the help sensor, the LED get ON when person present within the sensor range and pump get started when seepage water more than the given limit to the float switch. In remote mode operation, the operator can ON the particular LED and Pump whenever required with the help of PLC programming common in PC or mobile. Things are becoming more simple and easier for everyone in the world today because of advancement of technology. The Automation system consisting of control and information unit that's going to reduce the requirement of human need. The proposed system going to result in the power saving in an instant. The automated gallery lighting system provides a solution for energy saving, this can achieve by sensing an approaching person using the proximity sensor and then switching ON particular LED set on that range. As the person passes away from the particular range of the sensor the LED light set disconnected automatically with time delay. Buy this lot of energy get saved. In the present system, automated gallery lighting system light gets turned on automatically whenever the person enters the particular PIR sensor range. In order to decrease the problems of large energy consumption in an existing gallery lighting system, an automatic control system of gallery lighting is proposed. With the help of this system, it can save $80 \%$ of the energy consumption of the existing. The primary distinction between Microcontroller and PLC is that the PLC can operate in different a condition such as dust, moisture, heat, cold etc and the facility for extension of input and output arrangements.
\end{abstract}

KEYWORDS: Drainage Gallery, Switching ON Particular LED \& Input and Output Arrangements

Received: Nov 27, 2017; Accepted: Dec 17, 2017; Published: Jan 06, 2018; Paper Id: IJEEERFEB20181

\section{INTRODUCTION}

Automation plays a significant role in the day to day life and world economy. Automation system, replacing the manual framework. The research work shows automatic control of the gallery lighting system in dam going to result in power saving to some extent. With the help automation, world economy, and daily experience decreasing rapidly day by day. Automatic systems are being preferred over the manual framework. While automation furnished human operators for apparatus will aid the clients in bulky prerequisites for work, 
mechanization extraordinarily abatements the requirement for human tangible Also mental prerequisites too. Basically, gallery lighting system is one of the important parts during maintenance of the dam work.By present gallery lighting system, most of the places are only controlled by manual, a control switch set the gallery lighting. It is a so called first generation of the original lighting control which is inefficient and a waste of manpower and energy. The length of the dam galley is around $1.5 \mathrm{~km}$ to $2 \mathrm{~km}$. the atmosphere of the gallery is humid in nature due to back storage water and some amount of water leakage inside the gallery due to this condition the designing the lighting system is difficult to overcome theseproblems with the proper selection of enclosure of the IP rating un important tank. Under this condition, the proper enclosure is required in order to increase the life of the equipment used for lighting purpose more overly IP67 or IP68 equipment are used and they are sealed with proper insulation.

\section{DRAINAGE GALLERY IN DAM}

- The first functions of a gallery system in a dam is, it allows for inspection of the dam regularly, to monitor the behaviur of the dam and to carry out remedial work if required.

- Collect the seepage water through the foundation.

- Use for drilling and grouting the foundations.

- Install various instruments in the dam to study its structure behaviur.

- Provide space for the mechanical and electrical equipment's inspection and maintenance. Grouting usually is done to reduce the amount of seepage to the drain system, but it cannot in itself control uplift pressure

- Lighting of galleries is providedto permit inspections, maintenance and drilling of additional drains if they are found to be necessary.

Because drainage galleries cause critical scheduling issues and weaken the overall quality of dams, they ought to be utilized judiciously. Another function of the foundation gallery is to provide a space for drilling holes for providing what is called grout curtain, which is nothing but a series of holes drilled in a line deep inside the foundation and filled with pressurized cement mortar and also determining the location for grout hole in the foundation of the gallery The purpose of providing these holes and injecting them with cement mortar is to create a barrier in the foundation rock at the heel of the dam (Figure 19) which will prevent leakage of water from the reservoir and thus reduce uplift pressure at the bottom of the dam.

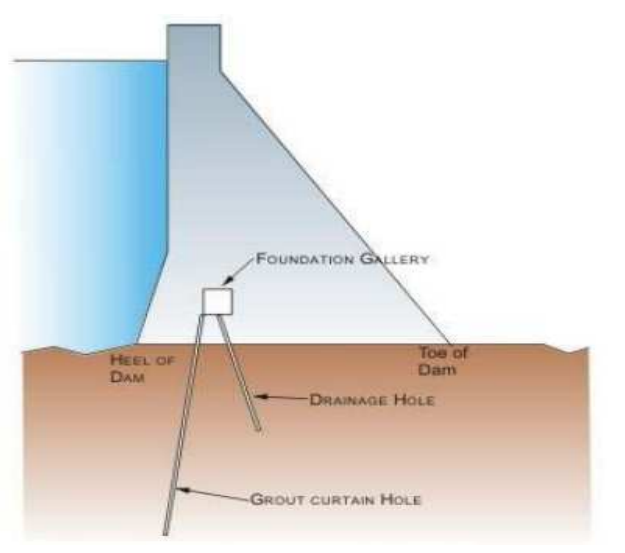

Figure 1: Drainage and Grout Curtain Holes 

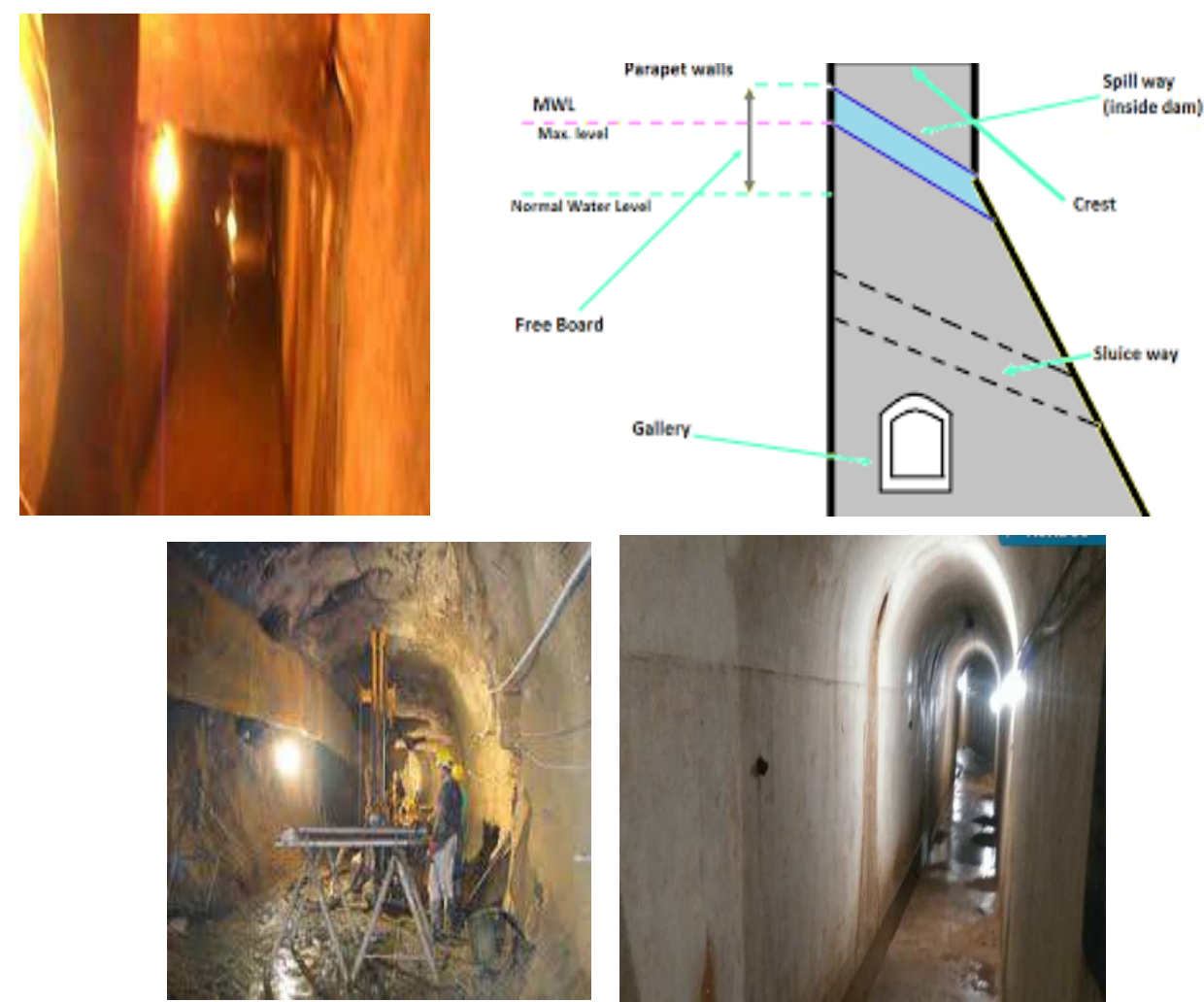

Figure 2: Gallery Lighting in Dam Using Incandescent Lamp System

Before designing lighting to any system, it is necessary to understand the nature of the surrounding environment. The atmospheric condition of gallery system is different, gallery temperature is very less and moisture content is more about this condition which IP rating component required should be studied. For this application, the particular IP rating waterproof LED strip is going to use. IP stands for ingress protection. For wet moisture content environment, there are two types IP rating LED strip is used IP67 and IP68 these both have some specification but different cost. An IP rating gives the information to end user of the item.

\section{What is IP?}

Every electrical and electronic component having an IP rating from cameras to phone and of course LED strip. IP rating explains the protection against waterproof, and level of protection provided against the dust interruptions, accidental contact with dust and water by mechanical casting and electrical enclosures. The first digit of IP rating varies from 0 to 6 . It explains the dust resistance and enclosure really made up of. 0 means not protect against dust and 6 means dust tight. The second digit in the IP rating varies from 0 to 9 and it explains the liquid ingress protection. Zero means not protect and 9 means protect against close range, high pressure and high temperature spray down. IP rating is the key factor for selecting the particular product for a particular application depending on the requirement.

\section{- IP67 Waterproof LED Light Strip}

IP67 rating is mainly used for outdoor lighting. In IP67 rating, 6 represent dust-tight protection and 7 indicates protection against water in destructive quantity. But it doesn't protect when the enclosure is submerged in the water for particular pressure and time. It protects against rain, just make ensure that LED strip not submerged into the underwater. 


\section{- IP68 Waterproof LED Light Strip}

Similarly, in IP68 rating 6 represents the ingress protection towards the dust-tight. 8 represent can that it is suitable for continuous immersion in water. It implies that the LED Strip can be immersed underwater in a particular amount of time because it is hermetically sealed.

\section{Programmable Logic Controller}

The programmable Logic Controller is more often than not found in the field near to the processing unit. PLCs are little and administrator interface may be simple as a button switch. In second era PLC providers have included simple in computerized change capability and provided adequate logic to arrange basic control loops [2] [3]. PLC is a hand-off substitution device and gives a reliable control to a stand-alone area of the process. PLC performs the all hands-off substitution capacities and to perform capacities like counting, timing and complex numerical applications. PLCs can perform PID, feed forward, and control work as well. PLCs can perform the work in DCS environment. The PLC system comprises five fundamental components:

- The PLC processor, or controller

- $\quad$ I/O (Input /Output) modules

- $\quad$ Memory Unit

- $\quad$ Power supply

- Programming software that runs on a PC

\section{In addition to these 5, most PLCs also have:}

\section{- A network interface}

PLCs have the capability to communicate with other devices. These devices incorporate computers running the programming program or collecting information approximately the fabricating prepare, a terminal that lets an operator enter commands into the PLC, or I/O are found in an inaccessible peat from the PLC. The PLC will communicate with the other devices through a network interface. Basic PLCs is builton a single printed circuit board. These units are by not expandable, the importance that extra inputs, outputs. Therefore, with couple exceptions, The point when utilizing this sort of PLC, the framework architect must fare the well should point out a unit that needs sufficient inputs, outputs, and modifying proficiency with handle both the display need of the framework What's more At whatever future adjustments that might a chance to be required.

These systems generally have an expansion port which will go to permits the addition of specialized units such as analogue input and output units or additional discrete inputs or outputs and high-speed counters. During model changeovers, PLCs were designed to eliminate the assembly line relays. This system operation of PLC is easier than the relay panels. This feature reduces the operational and installation cost of the control system compared with the electromechanical relay systems [4]. The basic block diagram of PLC system is as shown in Fig.1. 


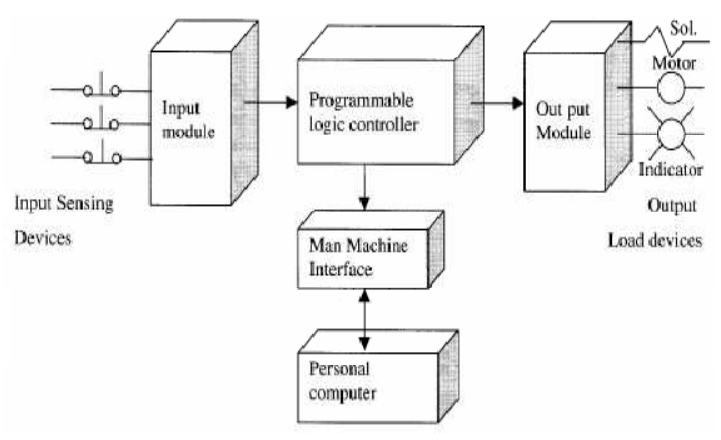

Figure 3: Basic Block Diagram of PLC System

\section{Block Diagram of Proposed System}

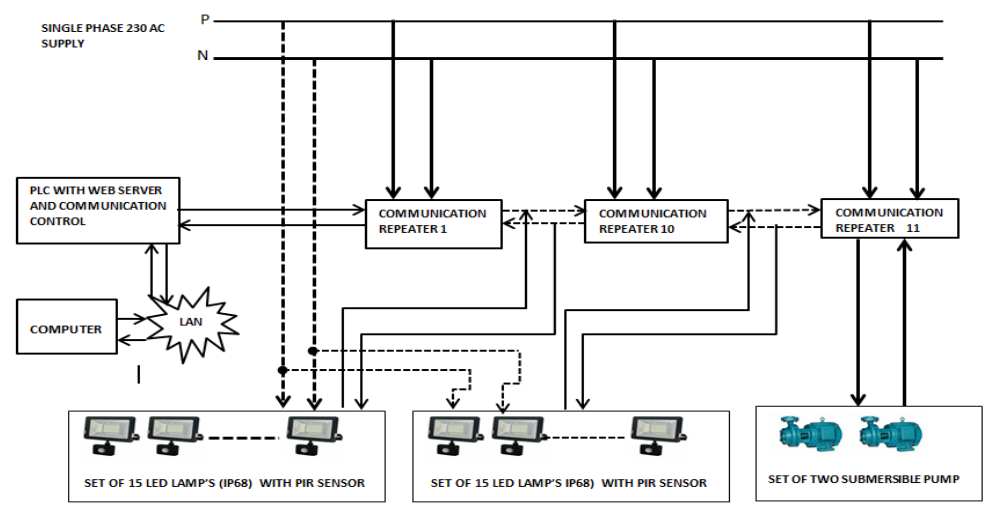

Figure 4: Block Diagram of the Proposed System.

The component required for implementing this project in the drainage gallery in the dam area

- Data cabling -4 core TEFLON coated 0.25 sq. mm wire with TEFLON coated outer sleeve with 1 pair 4pole IP67 waterproof connectors.

- LED lamps with PIR sensor battery backup controllers, full duplex mod bus interface and IP68 enclosures.

- Motor controllers with float switches and full duplex Modbus interface with IP68 enclosures

- PLC-ARM CPU based PLC with 7.5". Touch,screen user interface and remote connectivity/control via LAN/WLAN and built-in battery backup for 8 hours of continuous operation and mains power converter/charger.

- AC-DC convertors-24v DC, 60A, transformer type IP68 enclosure.

\section{METHODOLOGY}

Communication repeater is a Modbus communication repeater which is used to extend a number of nodes. PLC web server communication it interfaces to the system for monitoring or controlling purpose via the web-based server (network) connections. A web server is used to make the desired display or control pages available to the remote user or client and a data service act as an interface between the local and remote system (client) and handle exchanging data. For remote viewing of data and or web pages, the only requirement is a standard browser interface. In this system, the web server or data service device provides the connection between the machine or process and the internet. For installation, the connection to existing PLC or controller will require to extract data or enable control over the system The vast majority 
PLC's including items from ABB, Schneider Modicon and Siemens support the serial connection using communication protocols available from the PLC vendor or alternately outsider suppliers. Over a number situations, communication driver is available from the vendor as part of the embedded software application, whether those supplies employments a standard PLC protocol, or helps another open standard protocol for example, such that Modbus RTU that point the occupation for interfacing the slim server will be normally extraordinarily rearranged. with a LAN connection establishment, the remote system with its amplification software can now access the machine equipment as the thin server-assigned IP address via the factory network. With the help of ladder logic program, the system indicating the status of system components such as pilot light, relay contacts and motors applets can be written to represent and starting the motor and LED set with the click of a mouse. PLC is connected to the web server through the internet. The web server controls the interaction of the various system components in the real-time operating system and in editions of allocating tasks to the TCP/IP network security system in the form of a firewall is generally a part of the user's network infrastructure. Utilizing A standard ethernet interface the PLC links to the world wide web by means build-in-web server, permitting anyone with a PC and an internet connection to access information on PLC starting from anywhere around the world. With the help of Modem it is possible to access the control system from different locations, but generally, it is restricted to downloading and uploading data files and require a customized interface to get to control framework to giving any sort of control work between areas. The internet and world-wide-web offer a regular and effective receptive route to deliver data through hypertext links. A client-server framework gives each conclusion client the same interface with widespread get two administrations on the net combining a web browser and PLC provide users with a simple graphical means to access data and reset parameters from any pc attached to the world wide web.

Modbus: Modbus is a serial communication interface used for a multi-drop network based on the master/client architecture in the PLC. That first Modbus interface might have been free on deciding the unique Modbus interface around RS-485 on account of it permitted longer separation higher speeds and the possibility of a true multi-drop network. In simple terms, it is a method used for transmitting the information over serial lines between the electronic devices.

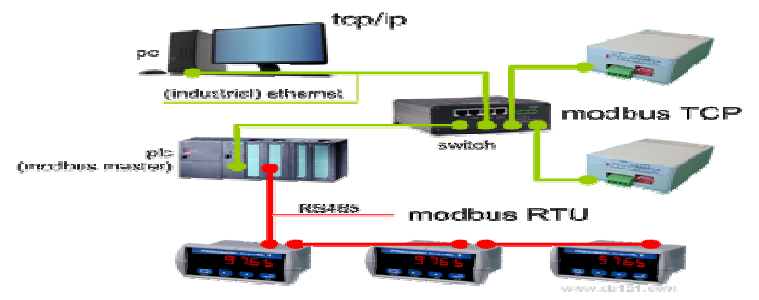

Figure 5: Modbus Interface

\section{- Communication Repeater}

An Inadigital communication system, a repeater is a device which receives a digital signal on an electromagnetic media, repeaters reduce divergence or cable loss in the system. With the help of series of repeaters, the data (information) can be transmitted over a long distance. It removes the unwanted noise in an incoming signal.

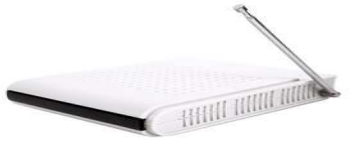

Figure 6: Communication Repeater 


\section{Flow Chart}

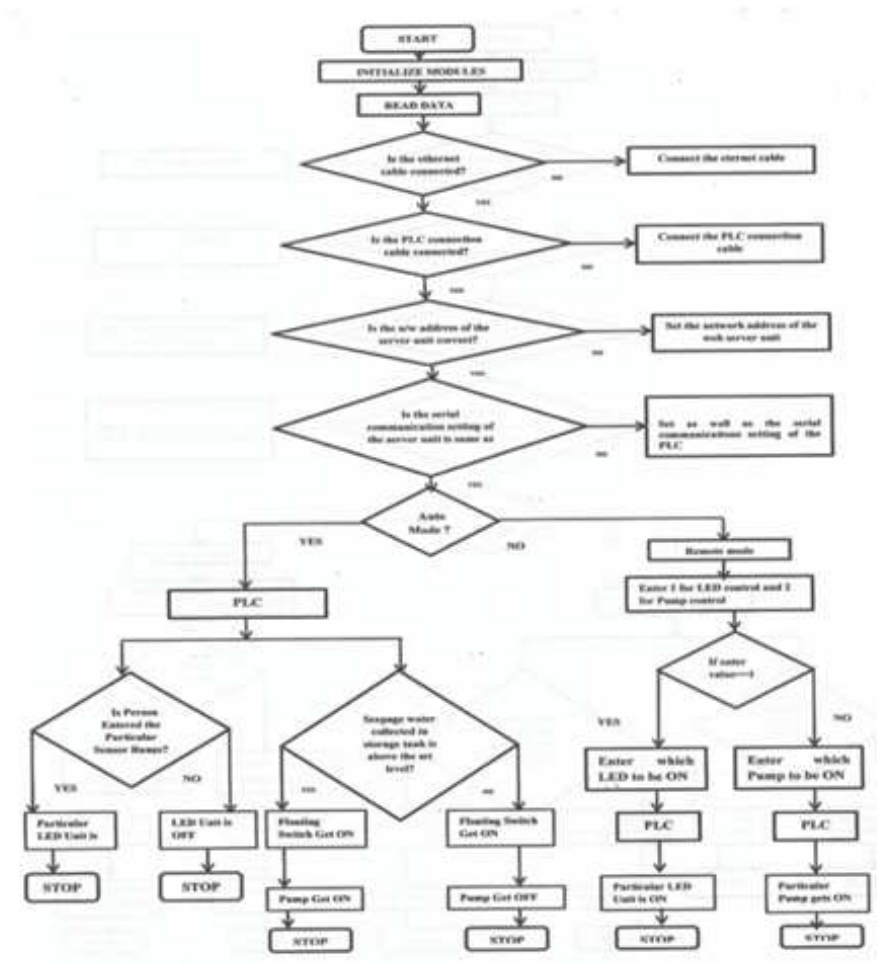

Figure 7: Flow Chart of Proposed System

PLCs are commonly selected for small discrete applications where high-level reliability and ruggedness is required. They offer high speed consecutive and logical control capabilities and are exceptionally great control arrangements for real-time applications. A PLC holds its working framework, client programs a little information in retentive (non-volatile) memory PLC system not only replaces the relay but also extended the logical capabilities of the system.

- $\quad$ PLCs connect PCs to the factory floor over the World Wide Web.

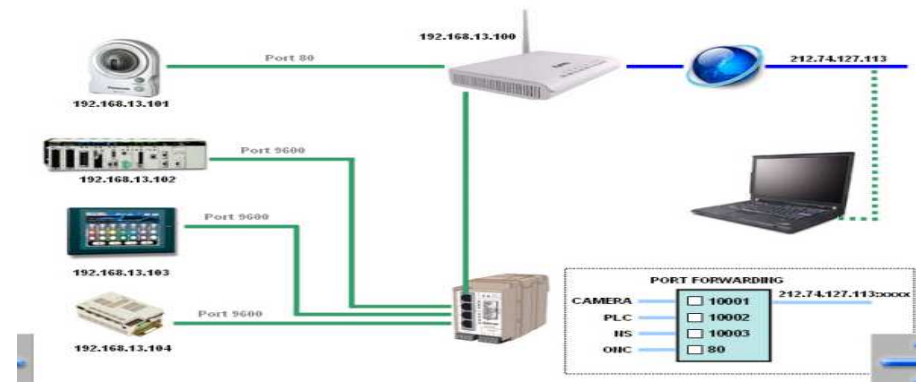

Figure 8: PLC with Web Server

In the proposed system the PLC integrated with embedded web server, PLC with web server consisting of full range input and output units to accept the snap-on communication adapter and this system consisting of Profibus, Modbus Plus, Ethernet networks and device Net. A ladder program is used to control the sensor units through the web server and the status of the system components such as pilot lights, relay contacts, and motors on the web page in the PC. Applets can be composed to demonstrate the start and stop boxes allowing the system for manual control for stopping and starting of 
the motor and LED light by clicking of a mouse on the PC. PLC is connected to the web server through the internet, in the real time system the web server control the various system components in addition to allocating tasks to the CPU. IP network provides the connection to the Ethernet driver. Utilizing a standard Ethernet interface, the PLC system is interfaced with World Wide Web through a built-in Web server, which going to permit the user to make changes in the PC and a Web association to get to the data on the PLC from anyplace around the world. In the past, inaccessible observing and control of industrial systems and forms took many forms. With the help of Modem, it became possible to access the control system from different locations, but they are generally restricted to downloading and uploading information records and require a customized interface to get to the control system. Moreover, providing any sort of control function between locations is maybe restricted. The Internet and the World Wide Web offer a common and effective open way to supply data through hypertext chains. A client-server framework gives each conclusion client the same interface with widespread access to organizations on the Net. Combining a Web browser and a PLC gives clients a straightforward, graphical implies to get to information and reset parameters from any PC associated with the World Wide Web. This makes it easier for users depending onthegraphical interfacing accessible to their desktop PC applications to disentangle the frustrating task of extricating usable data from austere PLCs. The Internet interface gives get to to the PLC by a client at a further area through the Web. Using this interface, the client can recover information such as PLC configuration, I/O and register status, working insights, diagnostics, and distributed I/O configurations. Overhauls to the operating computer program can too be downloaded through the Web. There is a particular advantage in recovering the data specifically from the PLC instep of through a PC network. PC-based Web servers require data travel between the PLC and the PC through the different systems in industrial facilities and plants. Information is at that point recovered from the PLC memory over the backplane and sent back through the same protocol levels. This makes it unsatisfactory to a timesensitive majority of the data. Embedding the Web server in the PLC ensures the auspicious flow of information needed on the factory floor. A Web server in the PLC has direct access to this information. At the same time, the built-in Ethernet interface grants this data to be effectively shared over the venture for fast decision making. Because PLC is embedded with Ethernet stack and the HTTP server, determinism is upheld both in the TCP/IP communications and in the PLC executive allowing it to be used to control I/O networks and synchronization between PLCs. As a result, the M1E executes 4,000 TCP/IP round-trip messages per second. To create a file system, the Web pages were assembled into a linked list and downloaded with the PLC executive to the M1E. The M1E trace the linked list chain until the requested filename is found in memory.

\section{THE FUTURE OF THIS SYSTEM}

Web-based PLCs can significantly reduce the plants run, downtime, and increasing productivity. A device equipped with an embedded Web server can monitor the proactively. In case it breaks or reaches certain pre-set parameters value's that indicate it is about to break, or comes to the certain preset value of the parameters, then it stops the system and it sends an alert message to the OEM or maintenance department, describing the particular problem, and requesting service. When breakdowns do occur, the Web-enabled equipment allows the OEMs who built it to remotely monitor and even repair their machines, reducing response time and expenses that ultimately are passed to the user, including staff time and travel expenses. Plant engineers can fix the issue within a factory over the Web from their office or even from home, eliminating those late-night trips to the plant. This same innovation permits plant engineers to at the same time screen different plants, making it conceivable to back up colleagues when they are out or on get-away or indeed to oversee different plants from a single location. Collecting plant data centrally permit business managers to assign new orders based 
on which plant can deliver with the quickest response time or the lowest cost of production of a particular point in time due to variations in utility rates, labor costs or other factors.

\section{- Lamp Unit}

The LED gallery light is an integrated light-emitting diode (LED) light fixture that is used for gallery light.

An LED gallery light is an integrated light that utilizes light emitting diodes as its light source. These are considered coordinates lights since in most cases, the lights and the installation are not isolated parts. In the modern manufacture system, the LED light cluster is sealed on a panel board and then assembled to the LED board with a heat sink to make an integrated lighting installation.

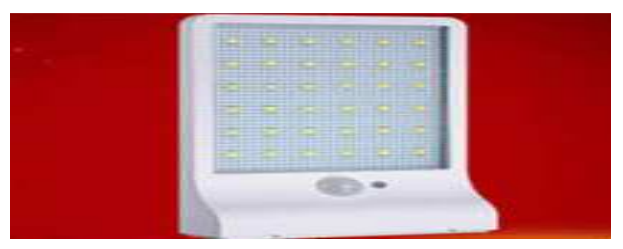

Figure 9: LED Unit

\section{Advantages of LED Gallery Lights}

- Low energy consumption: The much lower energy usage of LED lighting can reduce operating costs.

- Long and predictable lifetime: The lifetime of LED lights is usually 10 to 15 years.

- Quick turn on and off: Unlike fluorescent lamps, which take time to heat up once switched on, LEDs come to full brightness instantly

\section{- $\quad$ Sensor Unit}

It consists of the infrared sensor. It sends the message to other units when the motion is detected in the particular range of the sensor. This unit is placed at many locations like electric poles, house gates and inside or outside of the door, to ensure that every light turns on before pedestrians or a person to notice the lights. An infrared sensor is an electronic instrument that is used to sense certain characteristics of its surroundings by either emitting or detecting infrared radiation. It is also capable of measuring the heat of an object and detecting motion. Key benefits of infrared sensors include low power requirements, simple circuitry, and their portable feature. Here we use a Passive Infrared (PIR) motion detector used to control an outdoor, automatic light. At PIR-based motion detector is used to sense movement of people, animals, or other objects.
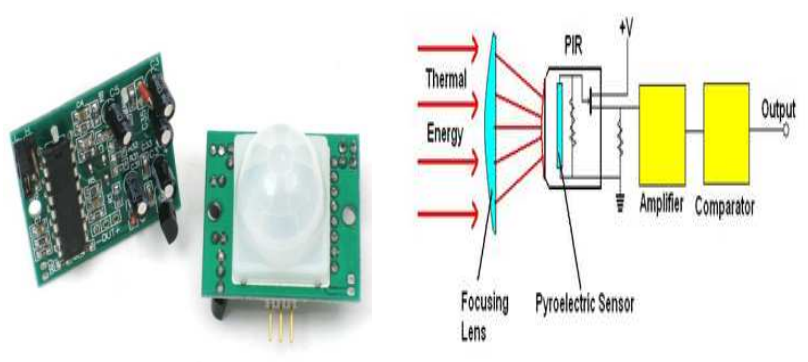

Figure 10: PIR Motion Sensor 


\section{CONCLUSIONS}

In this paper, an approach for controlling dam drainage gallery light system using PLC is proposed. The proposed controller gives fast, reliable and power efficient light switching. It also helps to reduce power consumption. The automated gallery lights look like normal lights, and the gallery lights will turn on before anyone sees them so that no one notices this light as Automated gallery Lights. This paper confirms that the proposed automated gallery lighting system based on sensors using PLC has great potential to revolutionize lighting system and in turn saves a large amount of energy. This proposed system continuously monitors the pump's which are used to pump the seepage water from the storage tank due to seepage. This system will remove the manual operation into automatic. This system operates in two modes i.e, remote mode and automated mode. In the automated mode with the help sensor, the LED get ON when a person present within the sensor range and pump get started when seepage water more than the given limit to the float switch. In remote mode operation, the operator can ON the particular LED and Pump whenever required with the help of PLC programming common in PC or mobile. Things are becoming more simple and easier for everyone in the world today because of advancement of technology

\section{REFERENCES}

1. Pantoni, R.P.; Brandao, D. "A geocast routing algorithm intended for street lighting system based on wireless sensor networks",, 2010 9th IEEE/IAS International Conference on Industry Applications (INDUSCON), On page(s): 1 - 6

2. Reluz Program (Jan. 2010). "National Program of Efficient Public Lighting and Traffic Lights". Available: http://www.eletrobras.gov.br/EM- Programas-Reluz.

3. Chen, P.-Y., Liu, Y.-H., Yau, Y.-T. and Lee, H.-C. (2008), "Development of a energy efficient street light driving system", IEEE International Conference on Sustainable Energy Technologies (ICSET 2008), Singapore, 24-27 November, pp. 761-4.

4. T.S. Huang and F. Luo: "Energy saving tunnel lighting system based on PLC", 2006 China International Conference on Electricity Distribution (CICED 2006), Beijing, China, pp. 527-533(2006) (in Chinese).

5. S. Nagai, S. Ishida, M. Shinji and K. Nakagawa: "Energy-saving lighting system for road tunnel", Underground Space Use: Analysis of the Past and Lessons for the Future, Istanbul, Turkey, pp. 625-631(2005)

6. R. Li, D. Fu, Z.Y. Chen: “Energy-saving \& Control of Tunnel Illumination”, Highway Engineering, Vol. 32, No. 5, pp. 204206(2007) (in Chinese). 